\title{
PROFILE OF IN-UTERO TRANSFERS IN YORKSHIRE \& HUMBER - EMBRACE TRANSPORT
} SERVICE

\author{
S. Babarao ${ }^{1}$, C. Harrison ${ }^{2}$, S. Hancock ${ }^{2}$ \\ ${ }^{I}$ Neonates, Leeds Teaching Hospitals NHS Trust, Leeds, ${ }^{2}$ Transport, Sheffield Children's Hospital, Sheffield, \\ $U K$
}

Background and aims: Embrace, combined paediatric and neonatal transport service for Yorkshire and Humber, facilitates in-utero transfers for a region with 75,000births/year. Our aim was to evaluate in utero transfer requests - indications for transfer, time spent locating cot/ bed, fibronectin use and outcomes.

Methods: Prospective audit of in-utero requests during 1/10/2010 - 7/01/2011. Data obtained via audit proforma and phone calls to maternity units.

Results: 86 transfers were facilitated out of 120 in-utero requests. The main reasons for requests were lack of neonatal cots $54 \%(65 / 120)$, maternal conditions requiring specialist care $2.5 \%(3 / 120)$, preterm gestation $24 \%(29 / 120)$ and impending preterm labour $7.5 \%(9 / 120)$.

$13 / 120$ of total requests - out of region cot located.

34 did not result in transfer despite cot/bed being located. The reasons were:

- Mother deteriorated/ went into labour $41 \%$

- Unit unwilling to accept out of region cot $12 \%$

- Local NNU arranged cot $15 \%$

- Referring hospital arranged transfer $6 \%$

- Changed decision re transfer need $20.7 \%$

Tertiary perinatal centres accepted $41 \%$ transfers.

Mean time facilitating transfers - 82 minutes.

Mean number of phone calls - 6.3 .

On average to organise 1 successful IUT, 2.1 maternity units and 3.4 neonatal units contacted.

$50 \%$ of mothers delivered within $24 \mathrm{hrs}$ of transfer.

$13.3 \%$ referrals used fibronectin.

Conclusion: IUT are a significant part of Embrace's workload. Increased use of fibronectin in impending deliveries would enhance appropriateness of transfers. 